\title{
A dimensional analysis approach to fatigue in quasi-brittle materials
}

\author{
Marco Paggi \\ Politecnico di Torino, Dep. of Structural and Geotechnical Engineering, Corso Duca degli Abrurzi 24, 10129 Torino, Italy \\ marco.paggi@polito.it
}

RIASSUNTO. Nel presente lavoro si propone uno studio di analisi dimensionale del fenomeno della fatica nei materiali quasi-fragili. Esso costituisce una generalizzazione della metodologia pionieristica proposta da Barenblatt e Botvina e si prefigge di interpretare le deviazioni dalle leggi di potenza classiche usate per caratterizzare il comportamento a fatica dei materiali. In base a questo approccio teorico, gli effetti dovuti alla dimensione microstrutturale (correlabile al contenuto volumetrico di fibre nei calcestruzzi fibrorinforzati), alla dimensione delle fessure e alla scala strutturale sulla legge di Paris e sulle curve di Wöhler sono discussi in un contesto matematico unificato. Il modello teorico è confermato dal confronto con rilevanti risultati sperimentali disponibili in letteratura, usati per determinare i valori degli esponenti di autosimilarità incompleta. Le informazioni fornite da questa teoria possono essere particolarmente utili per guidare la progettazione di nuovi esperimenti, dal momento che viene chiarito il ruolo delle diverse variabili adimensionalizzate che governano il fenomeno della fatica.

ABSTRACT. In this study, a generalized Barenblatt and Botvina dimensional analysis approach to fatigue crack growth is proposed in order to highlight and explain the deviations from the classical power-law equations used to characterize the fatigue behaviour of quasi-brittle materials. According to this theoretical approach, the microstructural-size (related to the volumetric content of fibres in fibre-reinforced concrete), the crack-size, and the size-scale effects on the Paris' law and the Wöhler equation are presented within a unified mathematical framework. Relevant experimental results taken from the literature are used to confirm the theoretical trends and to determine the values of the incomplete self-similarity exponents. All these information are expected to be useful for the design of experiments, since the role of the different dimensionless numbers governing the phenomenon of fatigue is herein elucidated.

KEYWORDS. Fatigue; Quasi-brittle materials; Fibre-reinforced concrete; Dimensional analysis; Incomplete selfsimilarity.

\section{INTRODUCTION}

$\mathrm{T}$ he assessment of the fatigue behaviour of quasi-brittle materials, such as plain or fibre-reinforced concrete (FRC), is particularly important from the engineering point of view. Concrete pavements for highways are in fact subjected to millions of cycles of repeated axial loads of high stress amplitude due to passing vehicles. Airport pavements are also subjected to a number of loading cycles during their design life, ranging from about several thousand to several hundred thousand. Concrete structures supporting dynamic machines may also fail due to repeated loadings causing complex stress states. To make the problem even more complex, very often failure is the result a steady decrease in the stiffness of the structural element, rather than by the propagation of a single macroscopic crack [1].

From the design point of view, the classical methods used to assess the fatigue performance of concrete are mainly empirical and are based on the cumulative fatigue damage approach, well-established for the analysis of the fatigue 
behaviour of metals [2,3]. Hence, the empirical S-N curve, relating the fatigue life or cycles to failure, $N$, to the applied stress range, $\Delta \sigma$ or $S$, is often used both for plain [4-6] and fibre-reinforced concretes [7-12]. A schematic representation of the Wöhler's diagram is shown in Fig. 1a, where the cyclic stress range, $\Delta \sigma=\sigma_{\max }-\sigma_{\min }$, is plotted as a function of the number of cycles to failure, $N$. In this diagram, we introduce the range of stress at static failure, $\Delta \sigma_{u}=\sigma_{\max }-\sigma_{\min }=\sigma_{u}-\sigma_{\min }=(1-R) \sigma_{u}$, where $\sigma_{u}$ is the material tensile strength, and we define the endurance or fatigue limit, $\Delta \sigma_{f l}$, as the stress range that a sample will sustain without fracture for $N_{\infty}=1 \times 10^{7}$ cycles, which is a conventional value that can be thought of as "infinite" life.

With the advent of fracture mechanics, a more ambitious task was undertaken, i.e., to predict, or at least understand, the propagation of cracks. Plotting the crack growth rate, $\mathrm{d} a / \mathrm{d} N$, as a function of the stress-intensity factor range, $\Delta K=K_{\max }-K_{\min }$, most of the experimental data can be interpreted in terms of a power-law relationship, i.e., according to the so-called Paris' law $[13,14]$. In spite of the fact that this approach requires a more elaborate testing procedure than for the S-N curves, it has also been applied to plain concrete [15-17] (see a schematic representation of the Paris' curve in Fig. 1b). Note that the power-law representation presents deviations for very high values of $\Delta K$ approaching $\Delta K_{\mathrm{cr}}=(1-R) K_{\mathrm{IC}}[18]$, where $K_{\mathrm{IC}}$ is the material fracture toughness, or for very low values of $\Delta K$ approaching the threshold stress-intensity factor range, $\Delta K_{\text {th }}$ [19]. Again, in close analogy with the concept of fatigue limit, the fatigue threshold is defined in a conventional way as the value of $\Delta K$ below which the crack grows at a rate of less than $1 \times 10^{-9} \mathrm{~m} / \mathrm{cycle}$.

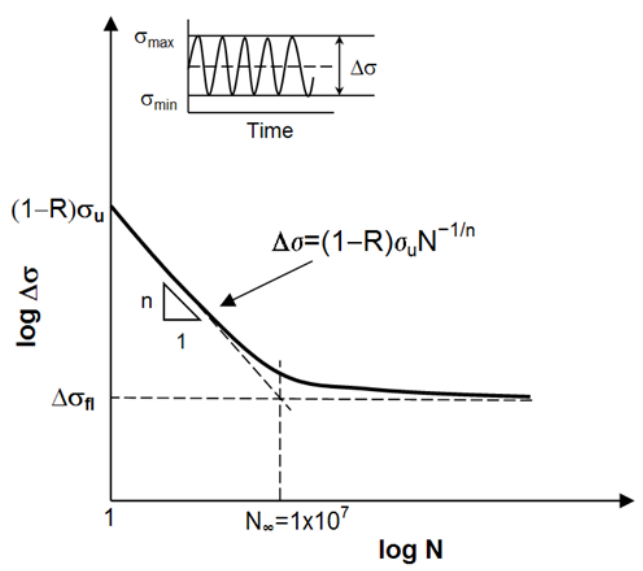

(a)

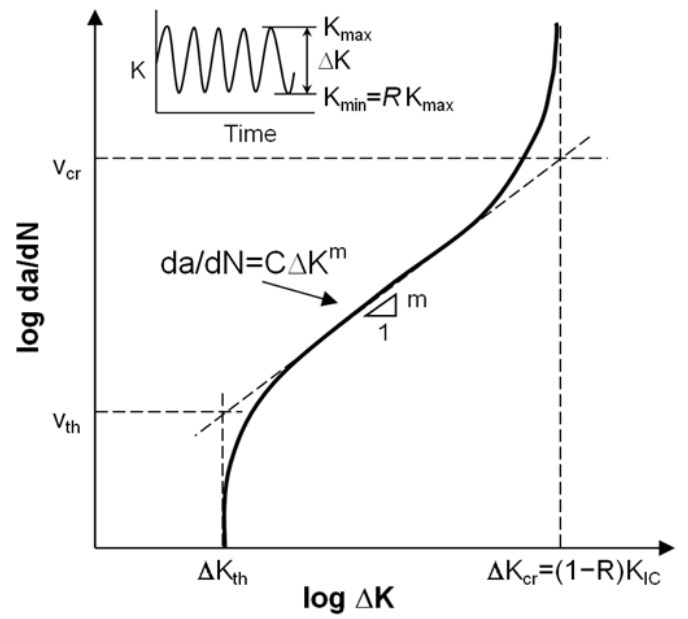

(b)

Figure 1: schemes of the (a) Wöhler and (b) Paris' curves with the related fatigue parameters.

Yet, the fatigue behaviour of quasi-brittle materials in bending or even in compression is not completely understood in terms of all the influential variables, such as the type of the loading cycle, cycling rate, structural size, crack length and, perhaps most important of all for FRC, fibres parameters. For instance, it has been experimentally shown in [15-17] that the coefficients entering the Paris' law depend on the material composition, on the structural size, and on the crack length, potentially explaining the large scatter in the reported values available in the literature. This result has also the fundamental implication that the Paris coefficients cannot be treated as true material constants. Similarly, the S-N curves are found to be dependent on the size of the tested specimen [11], on the loading conditions [1], as well as on the material composition [8].

From the modelling point of view, an important research effort towards simulating S-N curves of plain concrete and FRC in cyclic bending was put forward in $[11,20]$. This required the use of nonlinear fracture mechanics theories coupled with a damage law for modelling the degradation of the concrete cohesive crack stresses and of the fibres bridging in the process zone due to cyclic loading. However, in spite of the useful predictive capabilities of these models, their complex mathematical formulation limits their applicability to design purposes and confines them to the research environment. Moreover, although the damage model can be tuned on the basis of experimental data as proposed in [10], other choices for the expression of the damage variable are also possible (see e.g. [21]) and the connection existing between the evolution of damage and the outcome of the fatigue simulation has not yet been completely clarified. 
Following an independent line of research, the use of the concepts of dimensional analysis and incomplete self-similarity originally proposed by Barenblatt and Botvina [22,23] seems to be very promising in understanding the phenomenon of fatigue. Applying these concepts to metals, the present author has determined the relationship between the Paris' law parameters $C$ and $m$ on the basis of theoretical arguments [18]. Then, the Barenblatt and Botvina's analysis of the sizescale effects on the Paris' law parameter $m$ has been extended in [24] to the Paris' law parameter C. Finally, a dimensional analysis of the cumulative fatigue damage and of the damage tolerant approaches has been proposed in [25], interpreting the observed deviations from the Paris and Wöhler regimes within a unified theoretical framework. This approach promises to bring simplicity in what has been up to now considered as an unexplained set of data, putting into evidence the role of the most influential variables on the fatigue behaviour of materials.

The aim of the present study is to extend the dimensional analysis approach to quasi-brittle materials, determining the main dimensionless numbers influencing the $\mathrm{d} a / \mathrm{d} N-\Delta K$ and the $\mathrm{S}-\mathrm{N}$ curves. More specifically, the possibility of incomplete self-similarity in the dimensionless numbers related to the microstructural size, the crack size and the structural size is explored in details. As a result, different aspects that have always been so far treated separately are herein interpreted within a unified mathematical framework. The corresponding incomplete self-similarity exponents and their dependencies on the dimensionless numbers are also quantified on the basis of relevant experimental data taken from the literature. The new proposed correlations provide useful information for design purposes.

\section{GENERALIZED MATHEMATICAL REPRESENTATIONS OF FATIGUE}

\section{The generalized Paris law}

1 ccording to the pioneering work by Barenblatt and Botvina [22], the following functional dependence can be put forward for the analysis of the phenomenon of fatigue crack growth:

$$
\frac{\mathrm{d} a}{\mathrm{~d} N}=F\left(\sigma_{u}, K_{\mathrm{IC}}, \omega ; \Delta K, \Delta K_{\mathrm{th}}, h, d, a, E ; 1-R\right),
$$

where the governing variables are summarized in Tab. 1, along with their physical dimensions expressed in the LengthForce-Time class (LFT).

\begin{tabular}{|c|c|c|}
\hline Variable definition & Symbol & Dimensions \\
\hline Ultimate tensile strength & $\sigma_{u}$ & $\mathrm{FL}^{-2}$ \\
\hline Fracture toughness & $K_{\text {IC }}$ & $\mathrm{FL}^{-3 / 2}$ \\
\hline Frequency of the loading cycle & $\omega$ & $\mathrm{T}^{-1}$ \\
\hline Stress-intensity factor range & $\Delta K$ & $\mathrm{FL}^{-3 / 2}$ \\
\hline Threshold stress-intensity factor range & $\Delta K_{\text {th }}$ & $\mathrm{FL}^{-3 / 2}$ \\
\hline Stress range & $\Delta \sigma$ & $\mathrm{FL}^{-2}$ \\
\hline Fatigue limit & $\Delta \sigma_{f l}$ & $\mathrm{FL}^{-2}$ \\
\hline Structural size & $h$ & $\mathrm{~L}$ \\
\hline $\begin{array}{c}\text { Microstructural size } \\
\text { (aggregate or fibre size) }\end{array}$ & $d$ & $\mathrm{~L}$ \\
\hline Crack length & $a$ & $\mathrm{~L}$ \\
\hline Elastic modulus & $E$ & $\mathrm{FL}^{-2}$ \\
\hline Loading ratio & $R$ & - \\
\hline
\end{tabular}

Table 1: Governing variables of the fatigue crack growth phenomenon. 
Considering a state with no explicit time dependence, it is possible to apply the Buckingham's $\Pi$ Theorem [26] to reduce the number of parameters involved in the problem (see also [27-29] for the application to the quasi-static case). As a result, we have:

$$
\begin{aligned}
\frac{\mathrm{d} a}{\mathrm{~d} N} & =\left(\frac{K_{\mathrm{IC}}}{\sigma_{u}}\right)^{2} \Phi\left(\frac{\Delta K}{K_{\mathrm{IC}}}, \frac{\Delta K_{\mathrm{th}}}{K_{\mathrm{IC}}}, \frac{\sigma_{u}{ }^{2}}{K_{\mathrm{IC}}{ }^{2}} h, \frac{\sigma_{u}{ }^{2}}{K_{\mathrm{IC}}{ }^{2}} d, \frac{\sigma_{u}{ }^{2}}{K_{\mathrm{IC}}{ }^{2}} a, \frac{E}{\sigma_{u}} ; 1-R\right)= \\
& =\left(\frac{K_{\mathrm{IC}}}{\sigma_{u}}\right)^{2} \Phi\left(\Pi_{i}\right)
\end{aligned}
$$

where $\Pi_{i}(i=1, \ldots, 7)$ are dimensionless numbers. Note that $\Pi_{3}$ corresponds to the square of the dimensionless number $Z$ introduced by Barenblatt and Botvina [13] and to the inverse of the square of the brittleness number $s$ introduced by Carpinteri [17-19]. The number $\Pi_{5}$ was firstly considered by Spagnoli [8] for the analysis of the crack-size dependence of the Paris' law parameters. Here, it has to be noted that, according to Irwin, the ratio $\left(K_{\mathrm{IC}} / \sigma_{u}\right)^{2}$, which is used to made dimensionless the variables $h, d$ and $a$, is proportional to the plastic zone size, $r_{\mathrm{p}}$.

The number $\Pi_{4}$ is related to the microstructural length scale and has been introduced in [24] in order to interpret the effect of the grain size in metals on the coefficient $C$ of the Paris' law according to the experimental findings by Chan [30]. As far as plain concrete is concerned, this microstructural length scale should correspond to the average size of the aggregates. For FRC, the variable $d$ should correspond to the fibre diameter and Eq. (2) can be rewritten in order to put into evidence the fibre volumetric content. This parameter is computed as the ratio between the total area of fibres contained in a transversal cross-section of the specimen and the cross-section area of the specimen itself, $v_{f}=\left(A_{f} / A\right) \times 100=f(d / h)^{2}$. Here, the function $f$ represents a shape function which depends on the transversal cross-section of the fibres and on their amount. For a square specimen cross-section of side $b$ and for circular fibres we have $f=100 \times n_{f} \pi / 4$, where $n_{f}$ is the total number of crossed fibres. In this case, instead of considering the dimensionless representation (2), where all the variables with a physical dimension of a length are made dimensionless using the plastic zone size, it is more convenient to use the following alternative form:

$$
\begin{aligned}
\frac{\mathrm{d} a}{\mathrm{~d} N} & =\left(\frac{K_{\mathrm{IC}}}{\sigma_{u}}\right)^{2} \Phi\left(\frac{\Delta K}{K_{\mathrm{IC}}}, \frac{\Delta K_{\mathrm{th}}}{K_{\mathrm{IC}}}, \frac{\sigma_{u}{ }^{2}}{K_{\mathrm{IC}}{ }^{2}} h, \frac{d}{h}, \frac{\sigma_{u}{ }^{2}}{K_{\mathrm{IC}}{ }^{2}} a, \frac{l}{d}, \frac{E}{\sigma_{u}} ; 1-R\right)= \\
& =\left(\frac{K_{\mathrm{IC}}}{\sigma_{u}}\right)^{2} \Phi\left(\Pi_{i}\right)
\end{aligned}
$$

where the dimensionless number $\Pi_{4}$ has been replaced by $\Pi_{4}^{*}=\Pi_{4} / \Pi_{3}=d / h=\sqrt{v_{f} / f}$. Moreover, the number $\Pi_{6}=l / d$ (fibre length/fibre diameter) has also been suitably introduced in Eq. (3) and it corresponds to the fibre aspect ratio.

At this point, we want to see if the number of quantities involved in the relationships (2) or (3) can be reduced further from seven (or height). This can occur either in the case of complete or incomplete self-similarities in the corresponding dimensionless numbers. In the former situation, the dependence of the mechanical response on a given dimensionless number, say $\Pi_{i}$, disappears and we can say that $\Pi_{i}$ is non essential for the representation of the physical phenomenon. In the latter situation, a power-law dependence on $\Pi_{i}$ can be proposed, which usually characterizes a physical situation intermediate between two asymptotic behaviours.

Considering the dimensionless number $\Pi_{1}=\Delta K / K_{\mathrm{IC}}$, it has to be noticed that it rules the transition from the asymptotic behaviours characterized by the condition of nonpropagating cracks, when $\Delta K \rightarrow \Delta K_{\text {th }}$, to the pure GriffithIrwin instability, when $\Delta K \rightarrow \Delta K_{\mathrm{cr}}$. Moreover, incomplete self-similarity in $\Pi_{1}$ would correspond to a power-law 
dependence of the crack growth rate on the stress-intensity factor range, which is experimentally confirmed by the use of the Paris' law for concrete [15-17]. Therefore, complete self-similarity in $\Pi_{1}$ cannot be accepted and the assumption of incomplete self-similarity holds whenever $\Delta K$ is far lower than $K_{\mathrm{IC}}$, i.e., for $\Pi_{1}<<1$. As far as $\Pi_{3}$ is concerned, this number is expected to be particularly important in quasi-brittle materials, since it compares the structural size-scale $b$ with the plastic zone size. As regards $\Pi_{4}$ (or $\Pi_{4}^{*}$ for FRC), we know that, in metals, incomplete self-similarity is attained for $d / r_{\mathrm{p}} \cong 1$, i.e., for $\Pi_{4} \cong 1[25,30]$. A similar reasoning applies for $\Pi_{5}$, that is incomplete self-similarity in this number is expected when the crack length is comparable with the process zone size [17]. As regards $\Pi_{8}$, a dependence on $R$ is very often observed and therefore incomplete self-similarity in $\Pi_{8}$ is expected for $0<\Pi_{8}<1$.

Hence, assuming incomplete self-similarity in the dimensionless variables $\Pi_{1}, \Pi_{3}, \Pi_{4}, \Pi_{5}$ and $\Pi_{8}$, the following generalized representation of fatigue crack growth is derived starting from Eq.(3):

$$
\begin{aligned}
\frac{\mathrm{d} a}{\mathrm{~d} N} & =\left(\frac{K_{\mathrm{IC}}}{\sigma_{u}}\right)^{2}\left(\frac{\Delta K}{K_{\mathrm{IC}}}\right)^{\alpha_{1}}\left(\frac{\sigma_{u}^{2}}{K_{\mathrm{IC}}^{2}} h\right)^{\alpha_{2}}\left(\frac{v_{f}}{f}\right)^{\alpha_{3}}\left(\frac{\sigma_{u}^{2}}{K_{\mathrm{IC}}^{2}} a\right)^{\alpha_{4}}(1-R)^{\alpha_{5}} \Phi_{1}\left(\Pi_{2}, \Pi_{6}, \Pi_{7}\right)= \\
& =\Delta K^{\alpha_{1}} h^{\alpha_{2}} v_{f}^{\alpha_{3}} a^{\alpha_{4}}(1-R)^{\alpha_{5}} \frac{\Phi_{1}\left(\Pi_{2}, \Pi_{6}, \Pi_{7}\right)}{f^{\alpha_{3}} K_{\mathrm{IC}}^{\alpha_{1}+2 \alpha_{2}+2 \alpha_{3}+2 \alpha_{4}-2} \sigma_{u}^{2\left(1-\alpha_{2}-\alpha_{3}-\alpha_{4}\right)}}
\end{aligned}
$$

where the exponent $\alpha_{i}$ and, consequently, the dimensionless function $\Phi_{1}$, cannot be determined from considerations of dimensional analysis alone. Moreover, it is important to remark that the exponents $\alpha_{i}$ may depend on the dimensionless numbers $\Pi_{i}$. Eq. (4) can be regarded as a generalized Paris' law (see the classical expression $\mathrm{d} a / \mathrm{d} N=C \Delta K^{m}$ superimposed to Fig. 1b), in which the main functional dependencies of the parameter $C$ have been now explicitated.

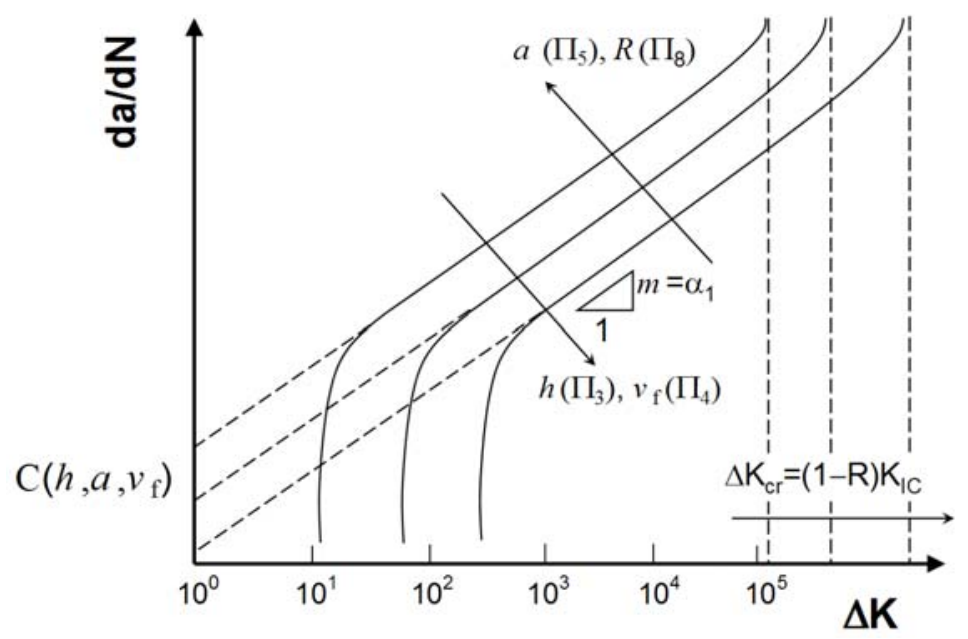

Figure 2: the effect of incomplete self-similarity in $\Pi_{3}, \Pi_{4}, \Pi_{5}$ and $\Pi_{8}$ on the Paris' curves.

In this mathematical framework, it emerges that the incomplete self-similarity exponent $\alpha_{1}$ simply corresponds to the Paris' law parameter $m$ that can be evaluated as the slope of the bilogarithmic $\mathrm{d} a / \mathrm{d} N$ vs. $\Delta K$ curve (see Fig. 2 ). Usually, $m$ varies from 5 up to 30 in quasi-brittle materials [31], a value particularly high as compared to metals, where $m$ ranges between 2 and 4 . For $\Delta K=1$, the intercept of the straight line with the vertical axis provides the logarithm of the multiplicative parameter $C$ (see Fig. 2). Under the assumptions of incomplete self-similarity discussed above, $C$ is no longer a true constant, but it is rather a power-law function of $h, v_{f}$ and $a$. Regarding the exponents $\alpha_{2}$ and $\alpha_{3}$, it is reasonable to expect them less than zero, with a reduction of $C$ by increasing either the size of the specimen or the amount of fibres. In fact, this would imply a shift of the vertical asymptote corresponding to $K_{\max } \rightarrow K_{\mathrm{IC}}$ towards 
higher values of $\Delta K$. This trend is consistent with the common observation that the fracture toughness of concrete is an increasing function of the size of the tested sample [32] and of the fibre volumetric content [10]. On the contrary, short cracks are expected to grow faster than long cracks [19] and therefore the exponent $\alpha_{4}$ is expected to be positive valued. The same reasoning holds for the loading ratio.

It is important to note that the above scaling law has been derived by considering the stress-intensity factor range as the driving parameter for crack growth. However, in quasi-brittle materials, where the cohesive crack model is routinely applied to describe crack propagation, it is also possible to find numerical results relating the crack growth rate to the range of energy release rate, $\Delta G$. Power-laws of the Paris form relating the crack growth rate to $\Delta G$ still hold [33] and Eq. (1) could be replaced by the following expression:

$$
\frac{\mathrm{d} a}{\mathrm{~d} N}=F\left(\sigma_{u}, G_{\mathrm{F}}, \omega ; \Delta G, \Delta G_{\mathrm{th}}, h, d, a, E ; 1-R\right)
$$

where the fracture energy, $G_{\mathrm{F}}$, has been suitably introduced instead of the fracture toughness $K_{\text {IC }}$ and the existence of a threshold range of energy release rate, $\Delta G_{\text {th }}$, has been postulated. Hence, Eq. (3) would become:

$$
\frac{\mathrm{d} a}{\mathrm{~d} N}=\frac{G_{\mathrm{F}}}{\sigma_{u}} \Phi\left(\frac{\Delta G}{G_{\mathrm{F}}}, \frac{\Delta G_{\mathrm{th}}}{G_{\mathrm{F}}}, \frac{\sigma_{u}}{G_{\mathrm{F}}} h, \frac{d}{h}, \frac{\sigma_{u}}{G_{\mathrm{F}}} a, \frac{l}{d}, \frac{E}{\sigma_{u}} ; 1-R\right)
$$

where we recognize that the dimensionless number governing the size-scale effects is no longer equal to $1 / s^{2}=\sigma_{u} h / K_{\mathrm{IC}}{ }^{2}$ as in Eq. (3), but it is now represented by the inverse of the energy brittleness number, $1 / s_{\mathrm{E}}=\sigma_{u} h / G_{\mathrm{F}}$. However, it is possible to demonstrate that Eq. (6) implies Eq. (3) and viceversa. Therefore, the dimensionless representation in Eq. (3) is equivalent to that in Eq. (6) and no additional dimensionless numbers have to be introduced. Recalling the Irwin's relationship between the stress-intensity factor and the energy release rate, it is possible to recast Eq. (6) in terms of stress-intensity factors. In doing that, we note that the energy brittleness number $S_{\mathrm{E}}$ is equal to the stress brittleness number $s$ times the inverse of the dimensionless number $E / \sigma_{u}$ [34], i.e., $s_{\mathrm{E}}=s^{2} /\left(E / \sigma_{u}\right)$. Therefore, $S_{\mathrm{E}}$ is nothing but a linear combination of two dimensionless numbers already defined in Eq. (3). Hence, considering plane stress conditions, Eq. (6) can be rewritten as follows:

$$
\frac{\mathrm{d} a}{\mathrm{~d} N}=\frac{K_{\mathrm{IC}}^{2}}{E \sigma_{u}} \Phi\left(\frac{\Delta K}{K_{\mathrm{IC}}}, \frac{\Delta K_{\mathrm{th}}}{K_{\mathrm{IC}}}, \frac{E \sigma_{u}}{K_{\mathrm{IC}}{ }^{2}} h, \frac{d}{h}, \frac{E \sigma_{u}}{K_{\mathrm{IC}}{ }^{2}} a, \frac{l}{d}, \frac{E}{\sigma_{u}} ; 1-R\right)
$$

which can be further manipulated by replacing the Young's modulus with the tensile strength, since the value of the ratio between these two variables is already taken into consideration by the dimensionless number $E / \sigma_{u}$. Therefore, after such a substitution, Eq. (7) becomes identical to Eq. (3). The inverse implication is straightforward and can be gained by applying the inverse of the Irwin's relationship.

\section{THE GENERALIZED WÖHLER REPRESENTATION}

o far, the crack growth rate has been chosen as the main output parameter characterizing the phenomenon of fatigue crack growth. However, it is also possible to consider the number of cycles, $N$, as the parameter representative of fatigue. Following this alternative route, we postulate again the following functional dependence:

$$
N=F\left(\sigma_{u}, K_{\mathrm{IC}}, \omega ; \Delta \sigma, \Delta \sigma_{f l}, h, d, a, E ; 1-R\right)
$$

where the definition of the governing variables is provided in Tab. 1. Considering a state with no explicit time dependence, it is possible to apply the Buckingham's П Theorem [26] to reduce the number of parameters involved in the problem: 


$$
N=\Psi\left(\frac{\Delta \sigma}{\sigma_{u}}, \frac{\Delta \sigma_{f l}}{\sigma_{u}}, \frac{\sigma_{u}^{2}}{K_{\mathrm{IC}}^{2}} h, \frac{d}{h}, \frac{\sigma_{u}^{2}}{K_{\mathrm{IC}}^{2}} a, \frac{l}{d}, \frac{E}{\sigma_{u}} ; 1-R\right)=\Psi\left(\Pi_{i}\right)
$$

where $\Psi$ is a dimensionless function. Note that Eq. (9) has been derived from Eq. (8) by choosing $\sigma_{u}, K_{\mathrm{IC}}$ and the plastic zone size as the main variables whose suitable combination provides a dimensionless number.

At this point, we want to see if the number of quantities involved in the relationship (9) can be reduced further from height. In close analogy with the procedure carried out for the Paris' law, we assume incomplete self-similarity in $\Pi_{1}$, $\Pi_{3}, \Pi_{4}, \Pi_{5}$ and $\Pi_{8}$ (the same conditions apply to the dimensionless numbers as in the derivation of Eq. (4)), obtaining:

$$
\begin{aligned}
N & =\left(\frac{\Delta \sigma}{\sigma_{u}}\right)^{\beta_{1}}\left(\frac{\sigma_{u}^{2}}{K_{\mathrm{IC}}^{2}} h\right)^{\beta_{2}}\left(\frac{v_{f}}{f}\right)^{\beta_{3}}\left(\frac{\sigma_{u}^{2}}{K_{\mathrm{IC}}^{2}} a\right)^{\beta_{4}}(1-R)^{\beta_{5}} \Psi_{2}\left(\Pi_{2}, \Pi_{6}, \Pi_{7}\right)= \\
& =\Delta \sigma^{\beta_{1}} h^{\beta_{2}} v_{f}^{\beta_{3}} a^{\beta_{4}}(1-R)^{\beta_{5}} \frac{\Psi_{2}\left(\Pi_{2}, \Pi_{6}, \Pi_{7}\right)}{f^{\beta_{3}} K_{\mathrm{IC}}{ }^{2\left(\beta_{2}+\beta_{3}+\beta_{4}\right)} \sigma_{u}^{\beta_{1}-2 \beta_{2}-2 \beta_{3}-2 \beta_{4}}}
\end{aligned}
$$

Eq. (10) represents a generalized Wöbler relationship of fatigue and encompasses the empirical S-N curves as limit cases. For instance, the S-N curve in Fig. 1a can be approximated by the Basquin power law:

$$
1 \times \Delta \sigma_{u}^{n}=N_{\infty} \Delta \sigma_{f l}^{n}=N \Delta \sigma^{n}=k
$$

where $\Delta \sigma_{u}$ is the range of stress at static failure, $\Delta \sigma_{f l}$ is the fatigue limit corresponding to a conventional fatigue life of $N_{\infty}=1 \times 10^{7}$ cycles, $\Delta \sigma$ is the stress range corresponding to a fatigue life $N$ and $k$ is a constant. Equating the first and the third terms in Eq. (11), we obtain he following power-law equation:

$$
N=\left(\frac{\Delta \sigma_{u}}{\Delta \sigma}\right)^{n}=\frac{(1-R)^{n} \sigma_{u}^{n}}{\Delta \sigma^{n}}
$$

Hence, the Basquin power law is predicted as a limit case of Eq. (10) when $\beta_{1}=-n, \beta_{2}=\beta_{3}=\beta_{4}=0$ and $\beta_{5}=n$.

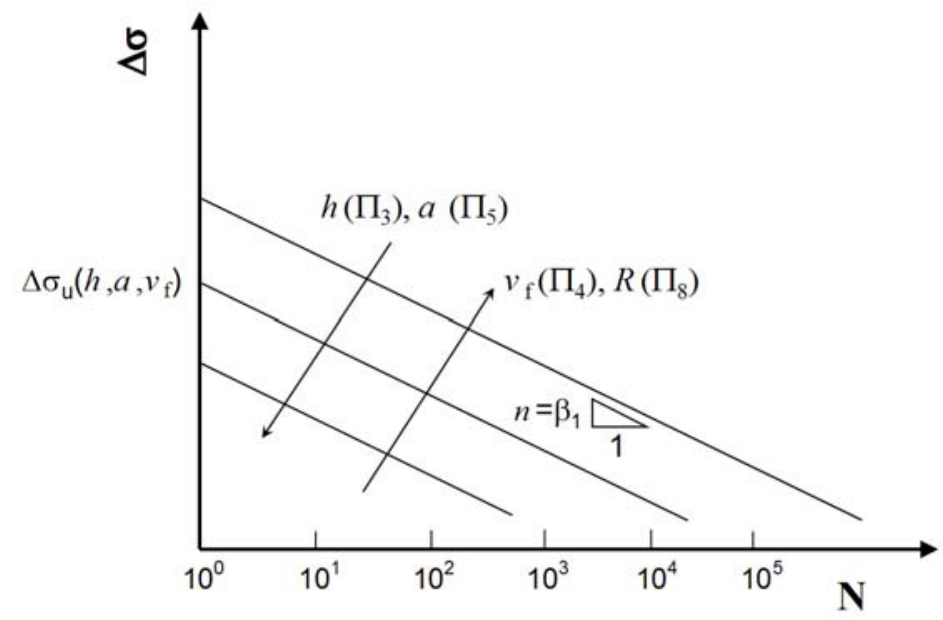

Figure 3: the effect of incomplete self-similarity in $\Pi_{3}, \Pi_{4}, \Pi_{5}$ and $\Pi_{8}$ on the S-N curves.

Therefore, in this framework, the incomplete self-similarity exponent $\beta_{1}$ simply corresponds to Basquin parameter $-n$ that can be evaluated as the slope of the $\Delta \sigma$ vs. $N$ curve in a bilogarithmic diagram (see Fig. 3). For $N=1$, the 
intercept of the straight line with the vertical axis provides the logarithm of the multiplicative parameter $\Delta \sigma_{u}$ (see Fig. 3). Under the assumptions of incomplete self-similarity discussed above, we expect $\beta_{2}$ less than zero, with a reduction of $\Delta \sigma_{u}$ by increasing the size of the specimen. Note that this trend is fully consistent with the well-known size-scale effects on the tensile strength of quasi-brittle materials [32] and with the recent findings in [35]. Similarly, we expect $\beta_{4}<0$ as for metals [36]. As regards the fibre volumetric content and the loading ratio, an opposite trend is expected, with $\beta_{3}$ and $\beta_{5}$ positive valued (see Fig. 3).

\section{APPLICATION TO QUASI-BRITTLE MATERIALS}

$\mathrm{I}$ $\mathrm{n}$ this section, the generalized Paris and Wöhler representations of fatigue are applied to plain concrete and FRC, providing a dimensional analysis interpretation to some of the most relevant experimental trends found in the literature. More specifically, the effect of the microstructural size (fibre diameter), of the crack size and of the structural size are carefully discussed.

\section{The effect of the microstructural size}

As shown in Section Generalized mathematical representations of fatigue, the use of fibres is beneficial from the fatigue standpoint, since an increase in fibre diameter (or, equivalently, in volumetric content) corresponds to a decrease in crack growth rate for a given value of $\Delta K$ and to an increase in the cycles to failure for a given value of $\Delta \sigma$. The quantify such an effect, the experimental flexural fatigue data by Johnston and Zemp [8] can be profitably used. They tested square concrete beams in cyclic bending without fibres or with smooth wire fibres with different volumetric contents $\left(v_{f}=0.5\right.$, 1.0 and $1.5 \%)$. All the fibres have an aspect ratio $l / d=75$.

The original S-N results are reported in the bilogarithmic diagram of Fig. 4, along with the best-fitting power-law regression curves. According to Eq. (10), the exponent of $N$ corresponds to $1 / \beta_{1}$ and we note that it is almost independent of the fibre volumetric content, ranging from -0.0218 to -0.0249 . For this type of fibres, the average value of $\beta_{1}$ is then equal to -43 . On the other hand, the multiplicative coefficient of the variable $N$ corresponds to $\Delta \sigma_{u}$ and this parameter is significantly affected by the presence of fibres. Plotting the values of $\Delta \sigma_{u}$ vs. the volumetric content in Fig. 5 and determining the best-fitting power-law equation, we find $\Delta \sigma_{u}=6.37 v_{f}^{0.26}$. According to Eq. (10) evaluated in correspondence of $N=1$, the exponent of $v_{f}$ is equal to $-\beta_{3} / \beta_{1}$, leading $\beta_{3}=-0.26 \beta_{1}=11.3$.

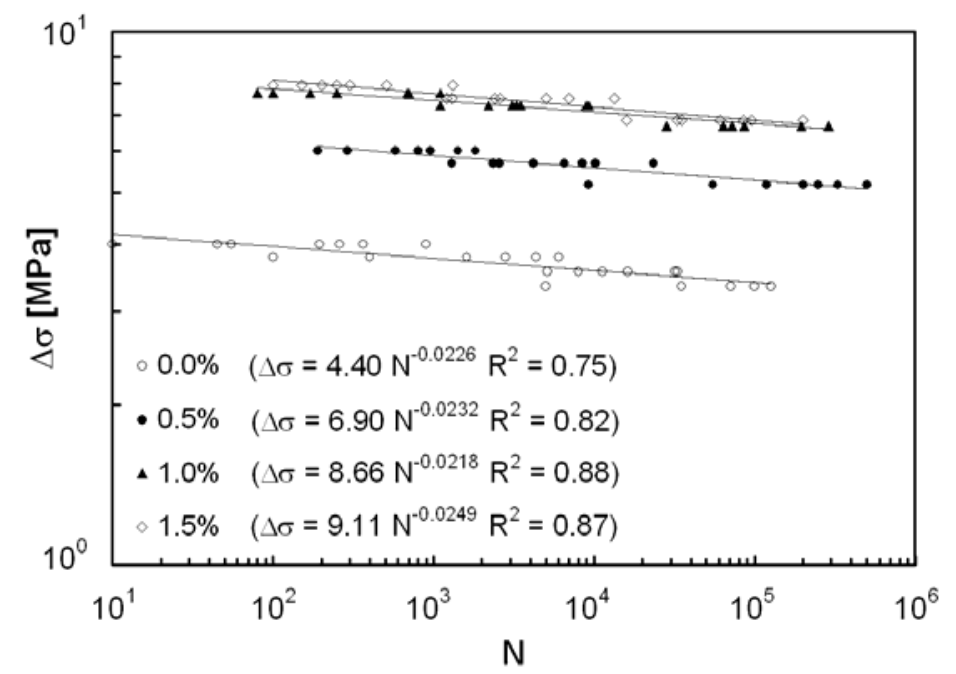

Figure 4: The effect of the fibre volumetric content, $v_{f}$, on the S-N curves as a result of incomplete self-similarity in $\Pi_{4}$ (experimental data from [8], aspect ratio $l / d=75$ ). 


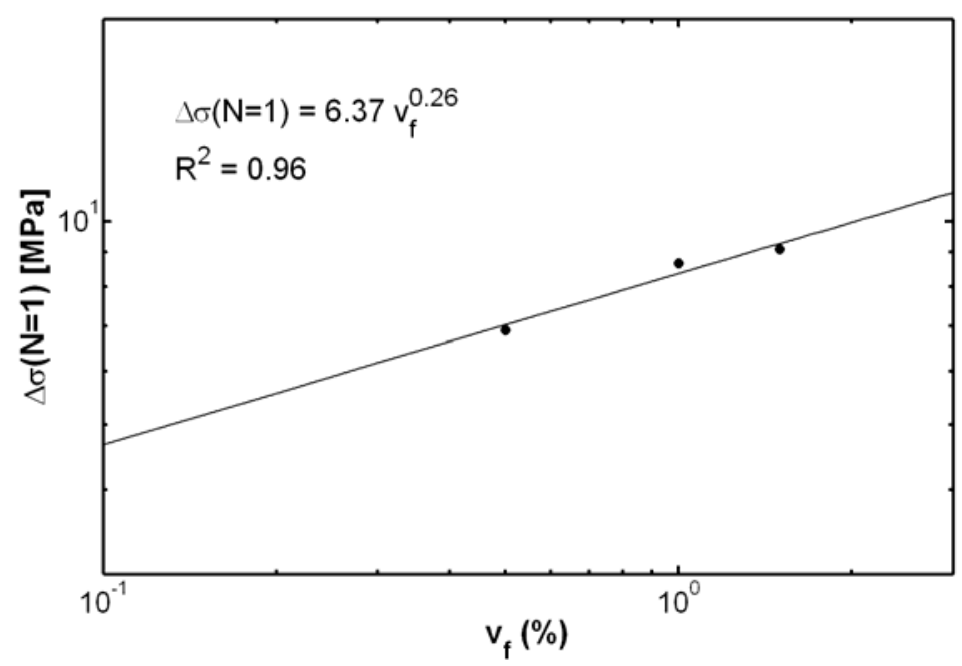

Figure 5: Determination of the incomplete-self-similarity exponent $\beta_{3}$.

As pointed out by Johnston and Zemp [8], the fibre aspect ratio has an important effect on the fatigue behaviour of FRC, whereas the fibre type (smooth wire, surface deformed wire, melt-extract and slit sheet) is of secondary importance. To show this effect in terms of dimensional analysis arguments, let us consider the experimental results by Singh et al. [12]. They tested FRC beams under fatigue loading with different volumetric content of fibres $\left(v_{f}=1.0,1.5\right.$ and $\left.2.0 \%\right)$. However, instead of using a single fibre type as in [8], 50\% of their total weight has an aspect ratio of $l / d=20$ and the remainder has an aspect ratio of $l / d=40$. Again, as for the previous case, the higher the volumetric content, the higher the fatigue life for a given applied stress range (see Fig. 6). However, now the S-N curves have an average slope equal to -0.030 , corresponding to an exponent $\beta_{1}=-33$. This value is significantly higher than -43 found in the case of a higher aspect ratio equal to $\Pi_{6}=l / d=75$. According to dimensional analysis, this difference has to be ascribed to the different value of the aspect ratio and to the fact that the power-law exponent $\beta_{1}$ depends on $\Pi_{6}$. From the engineering point of view, the use of longer fibres is clearly advantageous, since it increases the fatigue life of the specimen for a given applied stress range, reducing the exponent $\beta_{1}$.

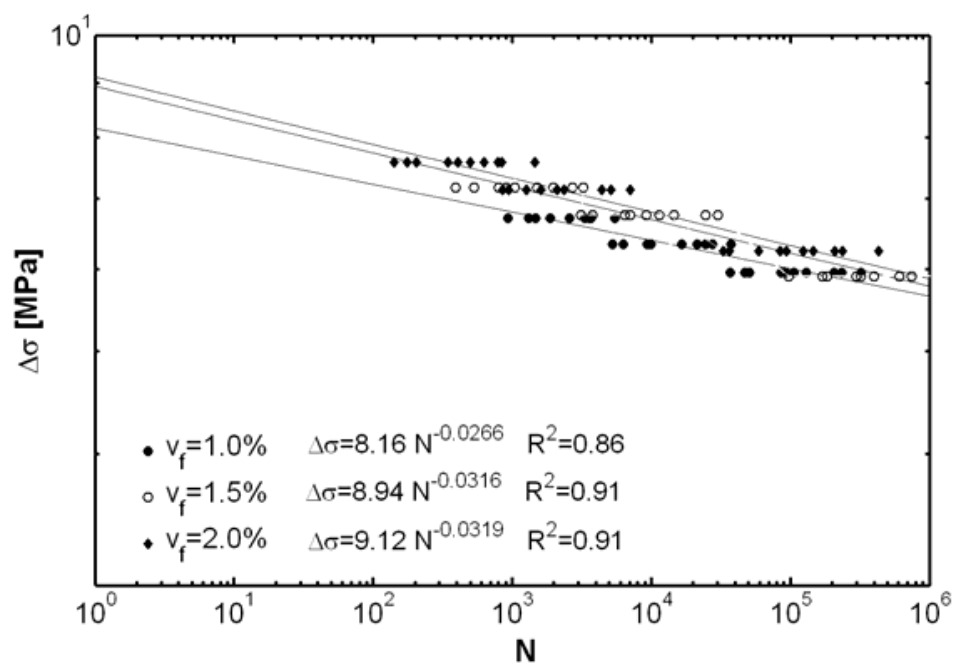

Figure 6: S-N curves of beams tested in flexural fatigue with $50 \%$ of fibres with $l / d=20$ and the remainder with $l / d=40$ (experimental data taken from [12]).

The effect of the crack size

Modified Paris' laws taking into account the effect of the crack size have been proposed both for metals and for quasibrittle materials. For metals, several researchers have questioned the validity of the similitude hypothesis, which states that 
"two different sized cracks embedded into two different sized bodies subjected to the same stress-intensity factor range should grow at the same rate". As a support to the theories against the similitude hypothesis, we mention the experimental results by Newman et al. [37], who observed that "in the threshold regime there is something missing in the (closure) model", and those by Forth et al. [36], revealing that similitude does not hold in Region I (the near-threshold region) and also in the lower portion of Region II. To overcome this problem, Molent et al. [39] and Jones et al. [40] have recently proposed a generalized Frost and Dugdale crack growth law, assuming that the crack growth rate is proportional to the accumulated plastic strain, averaged over a characteristic length ahead of the crack tip, $\mathrm{d} a / \mathrm{d} N=C^{\prime} a^{\left(1-m^{\prime / 2)}\right.} \Delta K^{m^{\prime}}$, where $C$ and $m$ are regarded as material constants. This equation states that $\mathrm{d} a / \mathrm{d} N$ is not only a function of the stressintensity factor range, but also of the crack length. Such a generalized Frost and Dugdale crack growth equation was successfully used to predict the growth of near micron sized cracks in both coupon and full scale aircraft fatigue tests and interpret a large amount of experimental data that could not be modelled using the Paris' law.

For concrete, a detailed experimental examination of crack propagation in flexural fatigue [17] has shown that the crack growth rate is not a monotonic increasing function of the crack length. For cracks shorter than the crack length at peak load in quasi-static monotonic loading, a deceleration stage was found, where $\mathrm{d} a / \mathrm{d} N$ is a decreasing function of $a$. Afterwards, an acceleration stage takes place and $\mathrm{d} a / \mathrm{d} N$ can be well approximated according to the classical Paris' law [17]. To model the deceleration stage, Kolloru et al. [17] proposed an empirical relationship between $\mathrm{d} a / \mathrm{d} N$ and the crack length, apparently independent of the Paris' law. Actually, it can be interpreted as a particular case of our proposed generalized Paris' law, simply allowing a crack-size dependence of the coefficient $C$, i.e., setting $C \propto a^{\left(n_{1}-n_{2} / 2\right)}$ $\left(\alpha_{4}=n_{1}-n_{2} / 2\right)$, where $n_{1}$ and $n_{2}$ are the power-law exponents for the two regimes found in [17].

\section{The effect of the structural size}

The effect of the structural size can be highlighted by considering the experimental data by Bažant and Xu [15] for normal strength concrete and by Bažant and Shell [16] for high strength concrete, subsequently re-examined by Spagnoli [41]. From their experimental results on self-similar beams tested in cyclic bending, the computed Paris' law exponent $m=\alpha_{1}$ was found to be dependent on the structural size. Plotting $m$ vs. $\Pi_{3}$ in Fig. 7 , we recognize that $m$ is a linear decreasing function of $\Pi_{3}$ for each type of concrete. The effect of the ultimate tensile strength is also important, since it affects the dimensionless number $\Pi_{7}=E / \sigma_{u}$. For normal strength concrete we have $\Pi_{7}=9686$, whereas for high strength concrete we have $\Pi_{7}=4303$ and the slope of the linear relationship between $m$ and $\Pi_{3}$ turns out to be an increasing function of $\Pi_{7}$ (see Fig. 7).

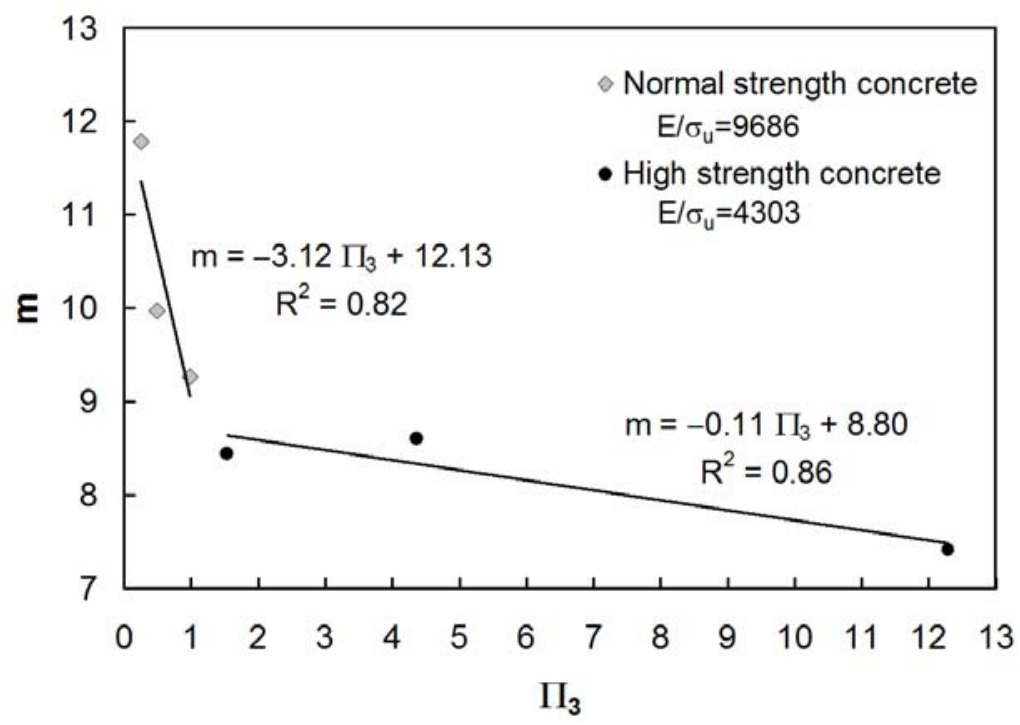

Figure 7: Size-scale effects on the Paris' law exponent $m=\beta_{1}$ for normal and high strength concrete (experimental data from $[15,16]$ reinterpreted in [41]). 
The experimental data by Bažant and Xu [15] for normal strength concrete and by Bažant and Shell [16] for high strength concrete can also be used to assess the hypothesis of incomplete self-similarity in $\Pi_{3}$ and therefore the size-scale effect on the parameter $C$. Plotting the Paris' law coefficient $C$ vs. $\Pi_{3}$ in a bilogarithmic diagram and computing the bestfitting power-law regression curves, we find $\alpha_{2}=4.7$ for normal strength concrete and $\alpha_{2}=1.1$ for high strength concrete (see Fig. 8).

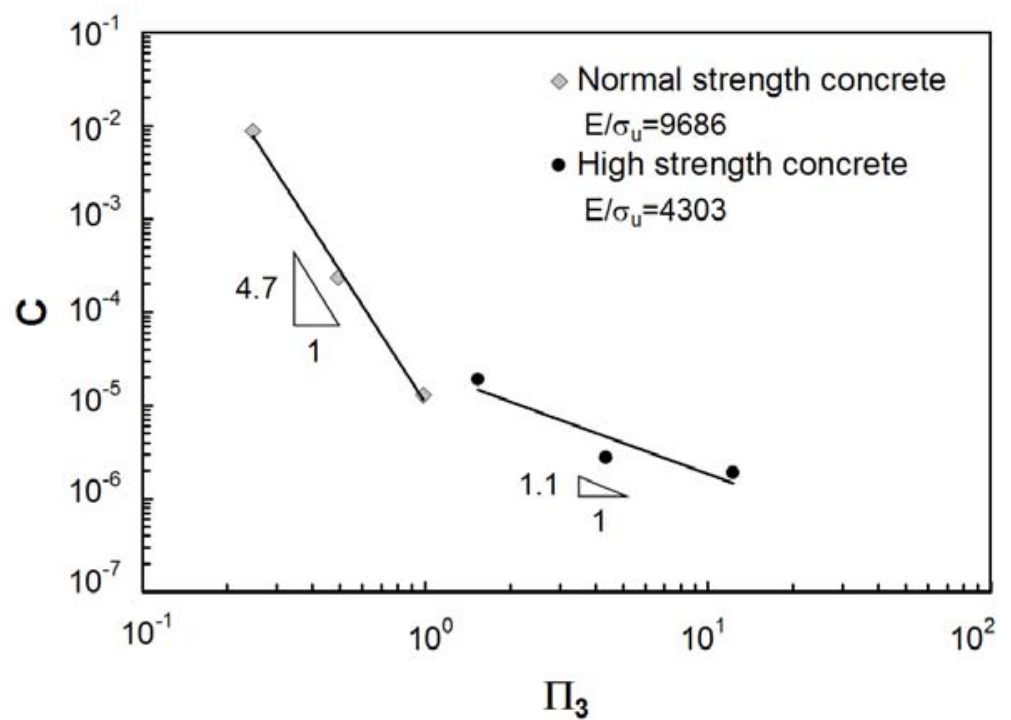

Figure 8: Size-scale effects on the Paris' law coefficient $C$ (evaluated using $\Delta K$ in $\mathrm{MPa} \sqrt{\mathrm{m}}$ and $\mathrm{d} a / \mathrm{d} N$ in $\mathrm{m} /$ cycle) as a result of incomplete self-similarity in $\Pi_{3}$ for normal and high strength concretes (experimental data from [15,16] reinterpreted in [41]).

Incomplete self-similarity in $\Pi_{3}$ is also expected to occur in the Wöhler regime, as recently put into evidence for metals in [35] on the basis of fractal concepts. For concrete specimens, the experimental data by Zhang and Stang [9] and Murdock et al. [42] show that the S-N curves corresponding to two different sizes are almost parallel to each other and translate vertically. In particular, the larger the beam size, the lower stress range at static failure (see Fig. 8). This result is in perfect agreement with the present theoretical predictions and with the numerical findings by Zhang et al. [11]. In this case, however, it is not possible to compute the incomplete self-similarity exponent $\beta_{2}$, since we have only two values of $\Delta \sigma(N=1)=\Delta \sigma_{u}$ for different beam depths.

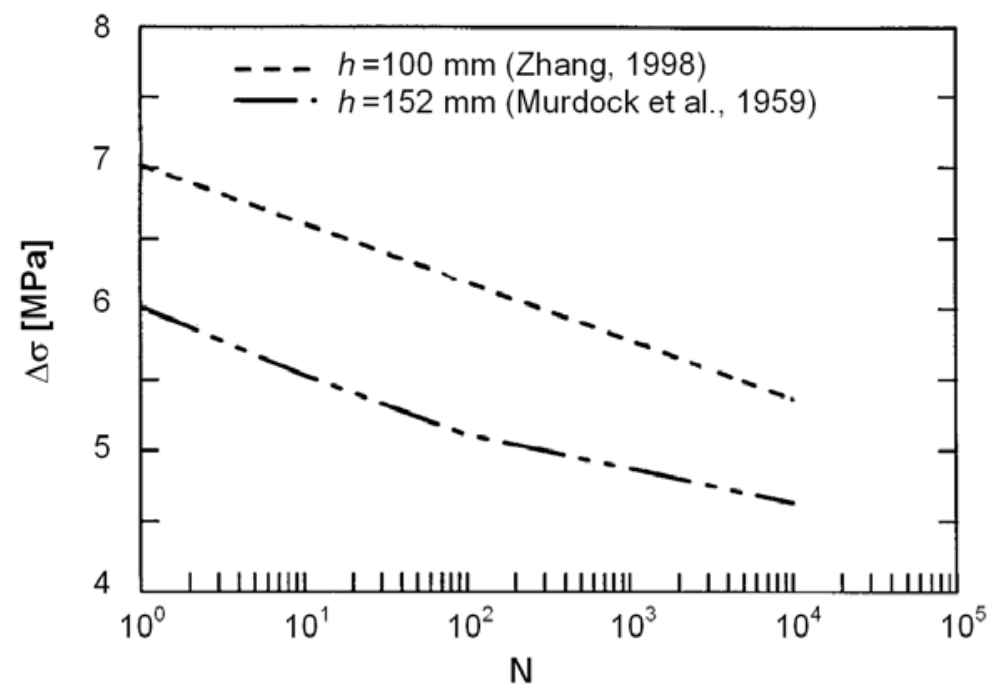

Figure 9: The effect of the structural size on the S-N curves (experimental data taken from [9,42]). 


\section{CONCLUSIONS}

I $\mathrm{n}$ the present paper, a generalized Barenblatt and Botvina dimensional analysis approach to fatigue crack growth has been proposed in order to highlight and explain the deviations from the classical power-law equations used to characterize the fatigue behaviour of quasi-brittle materials. It has been theoretically demonstrated that both the parameters entering the Paris' law and the Wöhler equation are microstructural-size, crack-size and size-scale dependent. From the theoretical point of view, these anomalous dependencies are due to incomplete self-similarities in the corresponding dimensionless numbers.

More specifically, as far as the Paris' law is concerned, it has been shown that the higher the structural size or the volumetric content of fibres, the lower the crack growth rate for a given stress-intensity factor range. Conversely, the higher the initial crack size or the loading ratio, the higher the crack growth rate. Regarding the S-N curves, the higher the structural size or the initial crack length, the lower the fatigue life for a given stress range. The opposite trend is noticed for the volumetric content of fibres and the loading ratio.

Finally, we have also shown that the slopes of the S-N curves are dependent on the fibre aspect ratio. Similarly, the slope of the Paris' curve is found to be dependent on the size of the specimen and on the ratio between the elastic modulus of concrete and its tensile strength. All these information are expected to be extremely useful for the design of experiments, since the role of the different dimensionless numbers governing the phenomenon of fatigue has been elucidated.

\section{REFERENCES}

[1] D. A. Hordijk, Heron, 37 (1992) 1.

[2] A. Wöhler, Z. Bauwesen (1860) 10.

[3] O. H. Basquin, Proc. ASTM, 10 (1910) 625.

[4] J. W. Murdock, C. E. Kesler, ACI J., 55 (1959) 221.

[5] R. Tepfers, ACI J., 76 (1979) 919.

[6] J. E. Butler, Strain, 26 (2008) 135.

[7] V. Ramakrishnan, G. Oberling, P. Tatnall, SP-105-13, ACI Special Publication, ACI, Detroit (1987) 225.

[8] C. D. Johnston, R. W. Zemp, ACI Mater. J., 88 (1991) 374.

[9] J. Zhang, H. Stang, ACI Mat. J., 95 (1998) 58.

[10] J. Zhang, H. Stang, V.C. Li, ASCE J. Mat. in Civ. Engrg., 12 (2000) 66.

[11] J. Zhang, V.C. Li, H. Stang, ASCE J. Mater. Civil Engng., 13 (2001) 446.

[12] S.P. Singh, Y. Mohammadi, S.K. Madan, J. Zhejiang University Science A, 7 (2006) 1329.

[13] P. Paris, M. Gomez, W. Anderson, 13 (1961) 9.

[14] P. Paris, F. Erdogan, J. Basic Eng. Trans. ASME, 58D (1963) 528.

[15] Z.P. Bažant, K. Xu, ACI Mater. J., 88 (1991) 390.

[16] Z.P. Bažant, W.F. Shell, ACI Mater. J., 90 (1993) 472.

[17] S.V. Kolloru, E.F. O’Neil, J.S. Popovics, S.P. Shah, ASCE J. Engng. Mech., 126 (2000) 891.

[18] Al. Carpinteri, M. Paggi, Engng. Fract. Mech., 74 (2007) 1041.

[19] M. Paggi, Al. Carpinteri, Chaos, Solitons and Fractals, 40 (2009) 1136.

[20] T. Matsumoto, V.C. Li, Cement \& Concrete Composites, 21 (1999) 249-261.

[21] K. L. Roe, T. Siegmund, Engng. Fract. Mech., 70 (2003) 209.

[22] G. I. Barenblatt, L. R. Botvina, Fat. Fract. Engng. Mater. Struct., 3 (1980) 193.

[23] G. I. Barenblatt, Scaling, Self-similarity and Intermediate Asymptotics. Cambridge: Cambridge University Press, (1996).

[24] M. Ciavarella, M. Paggi, Al. Carpinteri, J. Mech. Phys. Solids, 56 (2008) 3416.

[25] Al. Carpinteri, M. Paggi, Int. J. Fatigue, in press, doi: 10.1016/j.ijfatigue.2009.04.014

[26] E. Buckingham, ASME Trans., 37 (1915) 263.

[27] Al. Carpinteri, RILEM Mat. Struct., 14 (1981) 151.

[28] Al. Carpinteri, Engng. Fract. Mech., 16 (1982) 467.

[29] Al. Carpinteri, RILEM Mat. Struct., 16 (1983) 85.

[30] K. Chan, Scripta Metal. Mater., 32 (1995) 235.

[31] N. A. Fleck, K. J. Kang, M. F. Ashby, Acta Metall. Mater., 42 (1994) 365.

[32] Al. Carpinteri, Mech. Mater., 18 (1994) 89. 
[33] Y. Xu, H. Yuan, Proc. of the 12 $2^{\text {th }}$ International Conference on Fracture, Ottawa, Canada, (2009).

[34] Al. Carpinteri, In: Size-Scale Effects in the Failure Mechanisms of Materials and Structures (Proceedings of a IUTAM Symposium, Torino, Italy, 1994), Ed. A. Carpinteri, E \& FN SPON, London, (1996) 3.

[35] An. Carpinteri, A. Spagnoli, S. Vantadori, Int. J. Fatigue, 31 (2009) 927.

[36] J.C. Newman, Jr., E.P. Phillips, M.H. Swain, Int. J. Fatigue, 21 (1999) 109.

[37] J.C. Newman, Jr., A. Brot, C. Matias, Engng. Fract. Mech., 71 (2004) 2347.

[38] S.C. Forth, W.M. Johnston, B.R. Seshadri, Proc. 16 ${ }^{\text {th }}$ European Conf. Fracture, Alexandroupolis, Greece, (2006).

[39] L. Molent, R. Jones, S. Barter, S. Pitt, Int. J. Fatigue, 28 (2006) 1759.

[40] R. Jones, L. Molent, S. Pitt, Int. J. Fatigue 29, (2007) 1658.

[41] A. Spagnoli, Mech. Mat., 37 (2005) 519.

[42] J.W. Murdock, C.E. Kesler, ACI J., 55 (1959) 221. 